\title{
Tripartite motif containing 28 (TRIM28) promotes the growth and migration of endometrial carcinoma cells by regulating the AKT/mTOR signaling pathway
}

\author{
Yue Chen ${ }^{1,2, *}$, Haiyan Cheng ${ }^{2, *}$ and Heming Long ${ }^{1}$ \\ ${ }^{1}$ Department of Oncology, The First Affiliated Hospital of Gannan Medical College, Ganzhou City, Jiangxi Province, China \\ ${ }^{2}$ Department of Obstetrics and Gynecology, The First Affiliated Hospital of Gannan Medical College, Ganzhou City, Jiangxi \\ Province, China
}

\begin{abstract}
Although the medical science has been developed for decades, the molecular mechanism of endometrial cancer (EC) is not yet completely clear. Previous studies have shown that the tripartite motif containing 28 (TRIM28) plays a crucial role in tumor development. However, TRIM28 is rarely studied in EC, and its role and mechanism need to be further determined. This study was aimed to delve into the related molecular mechanism underling the role of TRIM28 in EC cell growth and migration. qPCR assays and Western blot assays revealed that the expression level of TRIM28 was higher in EC tissues or cell lines (HEC1B, AN3CA, and Ishikawa) than normal tissue or human endometrial epithelial cells (hEEC), respectively. Then, CCK-8 cell viability assay and clone formation assay were performed in HEC1B and AN3CA cell lines after overexpression or knockdown of TRIM28. The results verified that suppression of TRIM28 expression inhibited the proliferation of EC cells. The wound scratch healing assay and transwell assay were performed in HEC1B and AN3CA cell lines after overexpression or knockdown of TRIM28. The results showed that suppression of TRIM28 expression inhibited the invasion and migration of EC cells. Finally, the Western blot assays hinted that overexpression or knockdown of TRIM28 in HEC1B and AN3CA cell lines would promote or inhibit the phosphorylation of AKT and mTOR protein. These findings indicated that TRIM28 promoted the growth and migration of EC cells via regulating the AKT/mTOR pathway.
\end{abstract}

Key words: TRIM28 - Endometrial carcinoma - AKT - mTOR

Introduction

Endometrial cancer (EC) is an epithelial malignancy occurring in the endometrium, accounting for about $8 \%$ of all endometrial malignancies in women. In recent years, with the improvements in living standards, the rate of female obesity and incidence of EC has also been increasing (Samat et al. 2020). Patients with early EC generally have a good prognosis, with a 5-year survival rate up to 95\% (Miller et al. 2019), while those with advanced stage have a poor prognosis, with

\footnotetext{
* These authors contributed equally to this work.

Correspondence to: Haiyan Cheng, Department of Obstetrics and Gynecology, The First Affiliated Hospital of Gannan Medical College, No. 128, Jinling Road, Ganzhou City, Jiangxi Province, China E-mail: chenghaiyan0581@163.com
}

a low 5-year survival rate (Buhtoiarova et al. 2016). Although the medical science has been developed for decades, the molecular mechanism of EC is not yet completely clear. It has been reported that VEGF overexpression has been associated with EC progression. Meanwhile, PI3K-AKT-mTOR pathway and RAS/RAF/MEK pathway are also believed to be important factors in EC development. Therefore, it is of great significance to further explore the pathogenesis of EC and find new early diagnosis and treatment targets for improving the prognosis of EC.

Studies have shown that the human tripartite motif (TRIM) protein family can not only maintain the normal physiological function of the body, but also participate in the regulatory process of a variety of disease pathogenesis, including viral infection, tumorigenesis, neurodegeneration and other diseases (Czerwińska et al. 2017; Liu et al. 2019). TRIM28 is a large molecular protein with multiple 
domains and is a member of TRIM protein family. Among them, TRIM28 is highly expressed and plays an important role in many human malignant tumors (Yin et al. 2016). It has been reported that TRIM28 is overexpressed in breast cancer tissues and promotes the metastasis and proliferation of breast cancer cells (Wei et al. 2016). TRIM28 overexpression promoted the proliferation, metastasis and invasion of ovarian cancer cells by promoting $\mathrm{Wnt} / \beta$-catenin pathway (Deng et al. 2017). The down-regulation of TRIM28 can lead to the inhibition of cervical cancer cell cycle arrest and proliferation (Li et al. 2018). It is highly expressed in patients with gastric cancer, and its high expression is associated with poor prognosis (Yokoe et al. 2010). These results indicated that TRIM28 plays a crucial role in tumor development. However, TRIM28 is rarely studied in EC, and its role and mechanism need to be further determined.

In this study, it was found that TRIM28 was overexpressed in EC tissues and cells, and TRIM28 overexpression increased the migration and proliferation of EC cells by regulating the activity of $\mathrm{AKT} / \mathrm{mTOR}$ pathway. This study provides a new theoretical basis for the treatment of EC, and TRIM28/AKT/mTOR pathway may be a new molecular target for EC treatment.

\section{Materials and Methods}

\section{Tissue sample}

EC tissue and para-carcinoma tissue samples $(n=60$ in each group) were randomly collected at the First Affiliated Hospital of Gannan Medical College from EC patients. Informed written consents were obtained from all patients for sample collection. This study was approved by the Ethics Committee of the First Affiliated Hospital of Gannan Medical College and conducted according to the principles expressed in the Declaration of Helsinki (2013).

\section{Cell culture}

The human endometrial epithelial cells (hEEC) were obtained from Lonza (Basel, Switzerland). The human EC cell lines HEC1B, AN3CA, and Ishikawa were purchased from the Chinese Academy of Sciences (Shanghai, China). The cells were cultured in culture medium (RPMI-1640 or DMEM; Gibco, Carlsbad, CA, USA) with $10 \%$ fetal bovine serum (Gibco), and 1\% penicillin/streptomycin (Gibco) in a humidified $5 \% \mathrm{CO}_{2}$ incubator at $37^{\circ} \mathrm{C}$.

\section{$q P C R$}

Trizol reagent (Invitrogen, Carlsbad, CA, USA) was used for the extraction of total RNA from cells. The quantity and integrity of extracted total RNA were evaluated by a Nano Drop 1000 spectrophotometer (ThermoFisher Scientific, Waltham, MA, USA). The expression level of TRIM28 was detected through qRT-PCR by the SYBR Premix EX Taq (Takara, Dalian, China). The PCR primers were synthesized by Sangon Biological Engineering Technology (Shanghai, China). GAPDH was used as an endogenous reference gene to normalize mRNA expression levels. The relative expression level of TRIM28 was analyzed using the $2^{-\triangle \triangle \mathrm{Ct}}$ method. All reactions were performed in triplicates. Primer sequences are shown in Table 1.

\section{Western blot}

Briefly, cells were washed in pre-cooling PBS buffer three times, and the total protein was separated by RIPA (Beyotime, Shanghai, China). BCA protein assay kit (ComWin Biotechnology, Beijing, China) was used to detect the protein concentration. An equal amount of total proteins was electrophoresed by SDS-PAGE, and were transferred to the polyvinylidene difluoride membranes (PVDF; Millipore, Bedford, MA, USA) and blocked by 5\% non-fat milk for $1 \mathrm{~h}$. The protein was incubated with specific primary antibodies TRIM28 (Rabbit Anti-TRIM28 antibody, PA5-35079, 1:3000; Invitrogen), p-AKT (Rabbit Anti-AKT (phospho T308) antibody, ab38449, 1:3000; Abcam, Cambridge, MA, USA), AKT (Rabbit Anti-pan-AKT antibody, ab8805, 1:3000; Abcam), p-mTOR (Rabbit Anti-mTOR (phospho S2448) antibody, ab1093, 1:3000; Abcam), mTOR (Rabbit Anti-mTOR antibody, ab2732, 1:3000; Abcam), and GAPDH (Rabbit Anti-GAPDH antibody, ab9485, 1:3000; Abcam) overnight at $4^{\circ} \mathrm{C}$. Then, the membranes were further incubated with HRP-conjugated goat anti-rabbit immunoglobulin $\mathrm{G}$ secondary antibody (ab205718, 1:2000; Abcam) and the bands on the membranes were visualized by the ECL chemiluminescence reagent (Beyotime). The analyzed samples were normalized by GAPDH and the protein bands were quantified by gray value and analyzed by ImageJ software (National Institutes of Health, Bethesda, MD).

\section{Cell transfection}

For TRIM28 knockdown, two kind of human TRIM28-targeting short hairpin RNA (shRNA) oligonucleotide sequences and a scrambled shRNA as a negative control were cloned into vector. The shRNA sequences were shTRIM28\#1: CTGAGACCAAA CCTGTGCTTA and shTRIM28\#2: CCTGGCTCTGTTCTCTGTCCT (Su et al. 2018). The synthetic TRIM28-targeting shRNA, scrambled shRNA and TRIM28 mimics were obtained from GenePharma (Shanghai, China). After cells were cultured in 12-well plates for $24 \mathrm{~h}$, TRIM28targeting shRNA, scrambled shRNA and TRIM28 mimics were transfected into cells by Lipofectamine 3000 (Invitrogen). 


\section{CCK-8 cell viability assay}

To assess cell viability, a CCK- 8 assay was used. Briefly, $3.2 \times 10^{3}$ cells were plated into 96 -well plates in triplicates and treated with different conditions as indicated in each experiment. Following treatment, $10 \mu \mathrm{lCCK}-8$ solution (Beyotime) was applied and incubated for another $4 \mathrm{~h}$. The optical density value of each sample was checked at $450 \mathrm{~nm}$ through a microplate reader (BioTek, Winooski, VT, USA).

\section{Cell cloning assay}

To assess the extent of cell proliferation, a crystal violet staining method was used. Briefly, $4.5 \times 10^{3}$ cells were plated into 6 -well plates in triplicates and treated in different conditions as indicated in each experiment. After culture for 3 weeks, the supernatant was removed and $4 \%$ formaldehyde was added for $15 \mathrm{~min}$. Following treatment, cells were stained with $0.25 \%$ crystal violet solution for $25 \mathrm{~min}$.

\section{Scratch assay}

To assess the extent of cell migration, a scratch assay was used. Briefly, $4.5 \times 10^{5}$ cells were plated into 6-well plates in triplicates and treated in different conditions as indicated in each experiment. The next day, a horizontal line on the surface of the plate was scratched by a pipette tip and a ruler. Following treatment, the plate was softly washed three times with PBS and the cells were cultured in culture medium in a humidified $5 \% \mathrm{CO}_{2}$ incubator at $37^{\circ} \mathrm{C}$. The photos were taken $24 \mathrm{~h}$ after incubation.

\section{Transwell assay}

To assess the extent of cell invasion, a transwell assay was used. Briefly, $2.5 \times 10^{4}$ cells were plated into transwell inserts (Costar, Manassas, VA, USA) in triplicates and treated in different conditions as indicated in each experiment. $250 \mu \mathrm{l}$ of serum-free medium was added to the upper chamber and 550 $\mu \mathrm{l}$ of medium with $10 \%$ FBS was added to the lower chamber. After culture for $24 \mathrm{~h}$, cells that migrated to the lower surface of the membrane were fixed in $4 \%$ paraformaldehyde for $15 \mathrm{~min}$ and dyed by $0.1 \%$ crystal violet solution for $25 \mathrm{~min}$.

\section{Statistical analysis}

All data were shown as mean \pm standard error of the mean from three independent experiments. Comparisons between two groups were performed using Student's $t$-test. $p$ values of $<0.01$ (two-tailed) were considered to indicate a statistically significant difference. GraphPad Prism 5 (GraphPad Software Inc., San Diego, CA, USA) was used for data analysis.
A

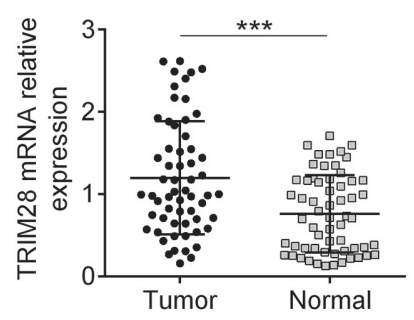

C

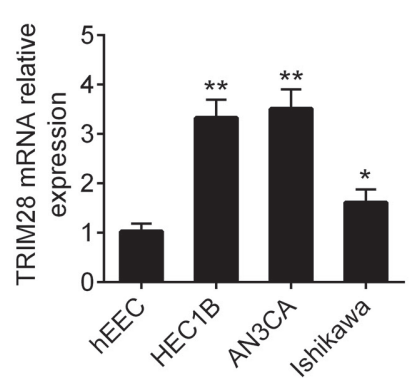

B

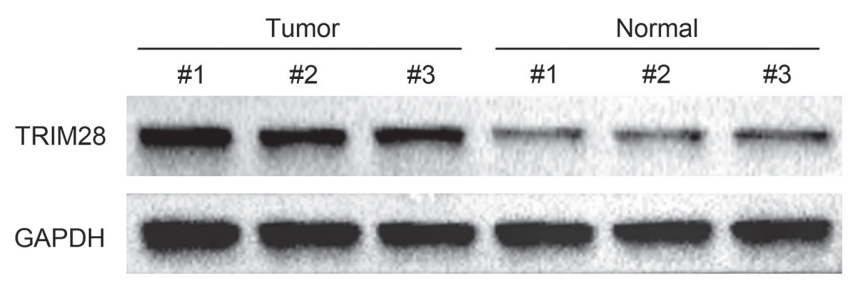

D

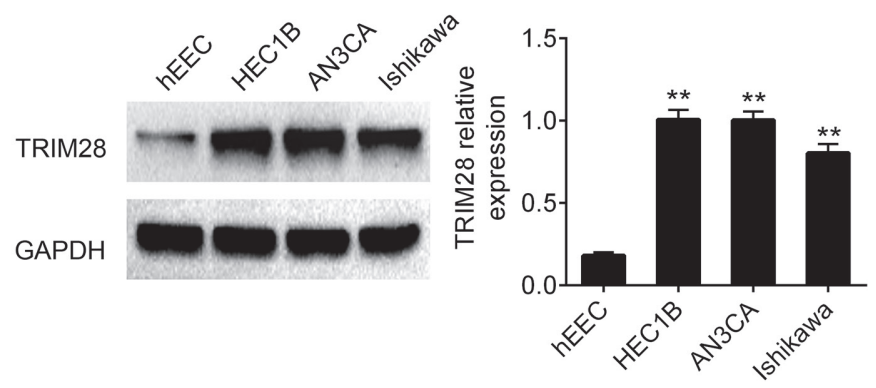

Figure 1. The mRNA expression level $(\mathbf{A} ; n=60)$ and the protein expression level $(\mathbf{B} ; n=3)$ of TRIM28 in EC (tumor) and normal tissues. The mRNA expression level (C) and the protein expression level (D) of TRIM28 in hEEC and three EC cell lines (HEC1B, AN3CA and Ishikawa). Data were presented as mean \pm SD with three independent experiments. ${ }^{*} p<0.05,{ }^{* *} p<0.01,{ }^{* *} p<0.001 v s$. Normal group or hEEC group. 


\section{Results}

\section{TRIM28 was overexpressed in EC tissues and cells}

In order to investigate the correlation between the expression levels of TRIM28 and EC progression, a total of $60 \mathrm{EC}$ tissue and normal tissue samples were recruited and analyzed by qRT-PCR and Western blot. The expression level of TRIM28 was obviously increased in EC patients compared with those in normal group (Fig. 1A,B). Then, the expression level of TRIM28 in human endometrial epithelial cells (hEEC) and three EC cell lines (HEC1B, AN3CA and Ishikawa) were also determined by qRT-PCR and Western blot. The results revealed that the expression level of TRIM28 was significantly higher in EC cell lines compared to that in hEEC cell line (Fig. 1C,D). These results verified that TRIM28 was overexpressed in EC tissues and cells.

\section{Suppression of TRIM28 expression inhibited the proliferation of EC cell}

To examine the effects of TRIM28 on EC cell proliferation, EC cell lines (HEC1B and AN3CA) were transfected with empty vector (Control), TRIM28 mimics (TRIM28),

A
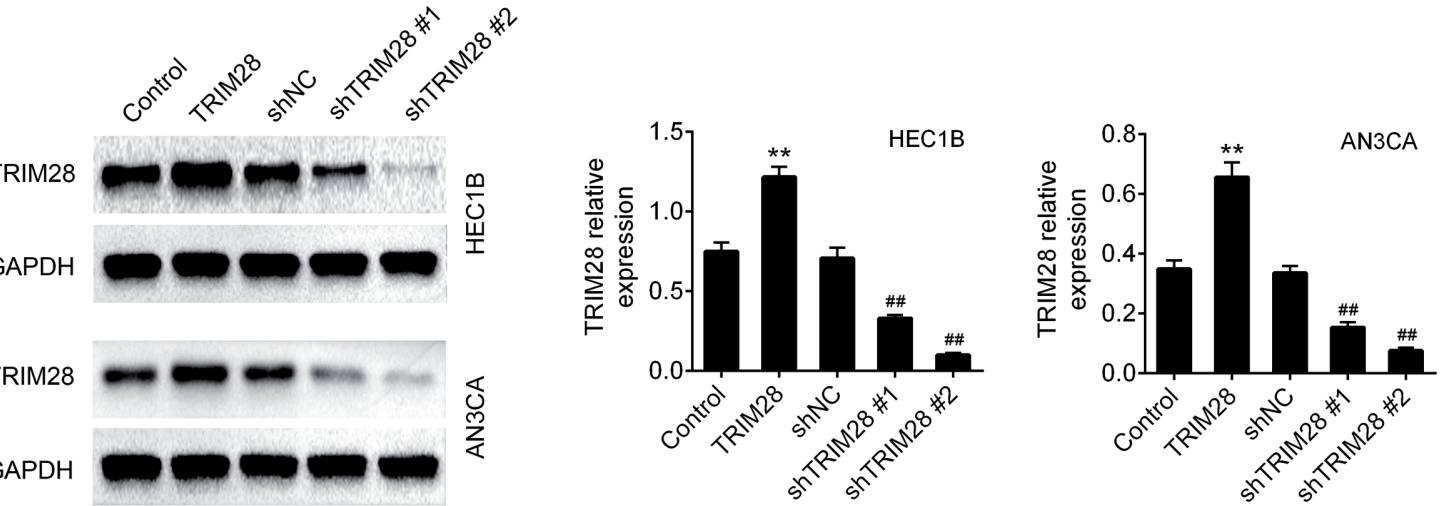

B

C
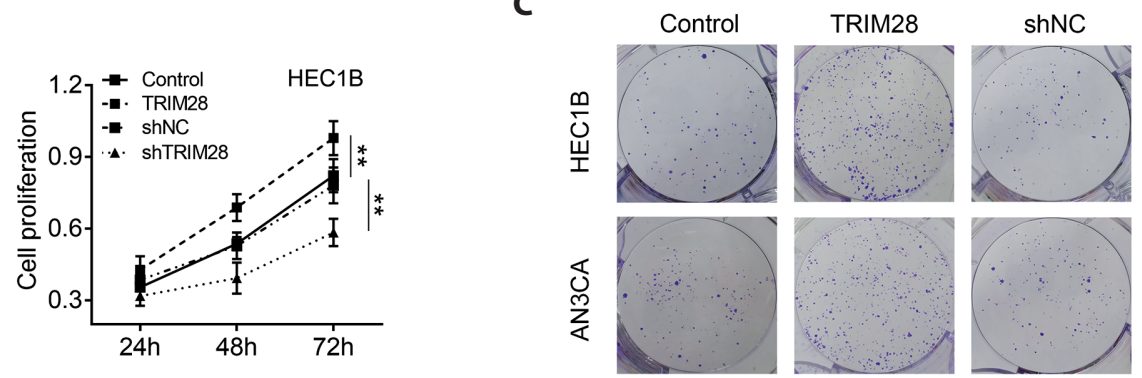

shTRIM28
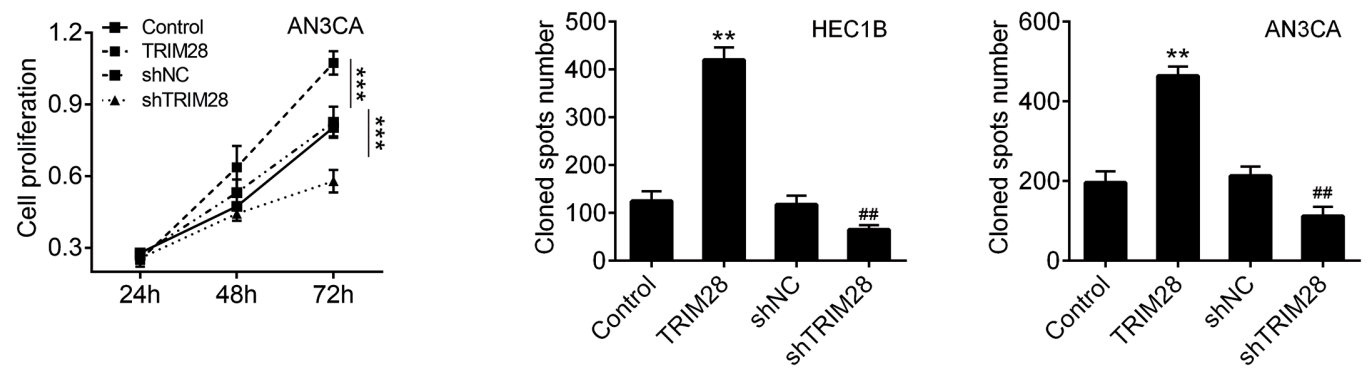

Figure 2. Suppression of TRIM28 inhibited the proliferation of EC cells. A. Overexpression or knockdown of TRIM28 in HEC1B and AN3CA cell lines was verified by Western blotting. B. CCK-8 cell viability assays detected the proliferation of HEC1B and AN3CA cell lines after overexpression or knockdown of TRIM28. C. Clone formation assay detected the proliferation of HEC1B and AN3CA cell lines after overexpression or knockdown of TRIM28. Data were presented as the mean \pm SD with three independent experiments. ${ }^{* *} p<$ $0.01,{ }^{* * *} p<0.001$ vs. Control group; ${ }^{\# \#} p<0.01$ vs. shNC group. 
A

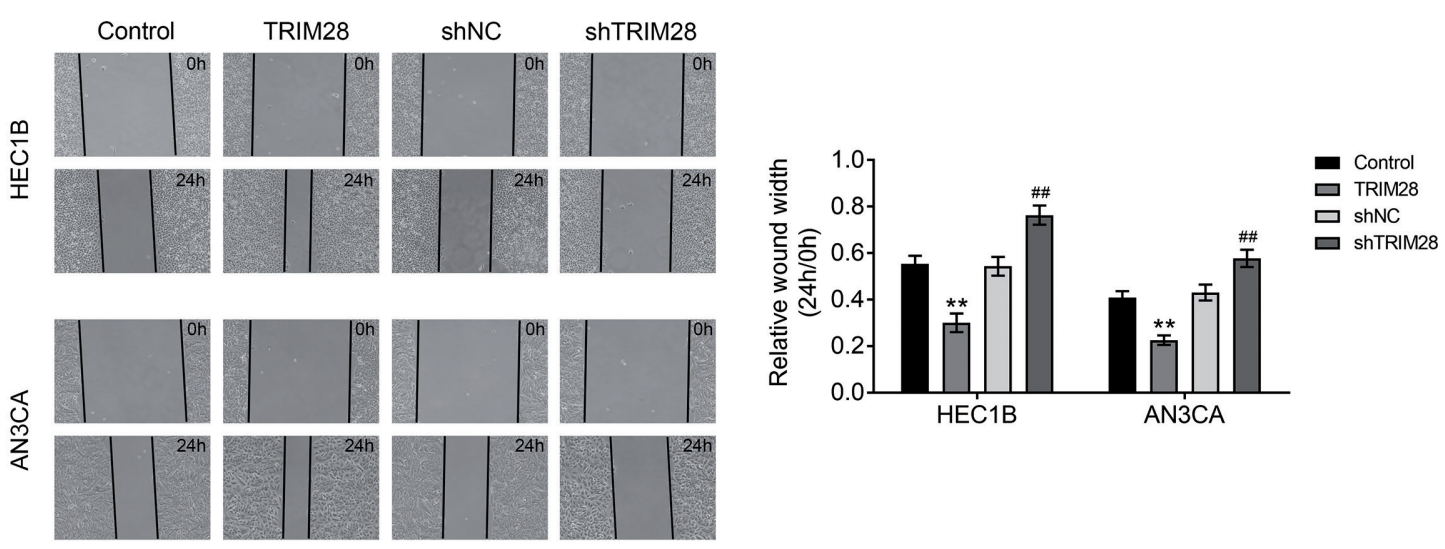

B
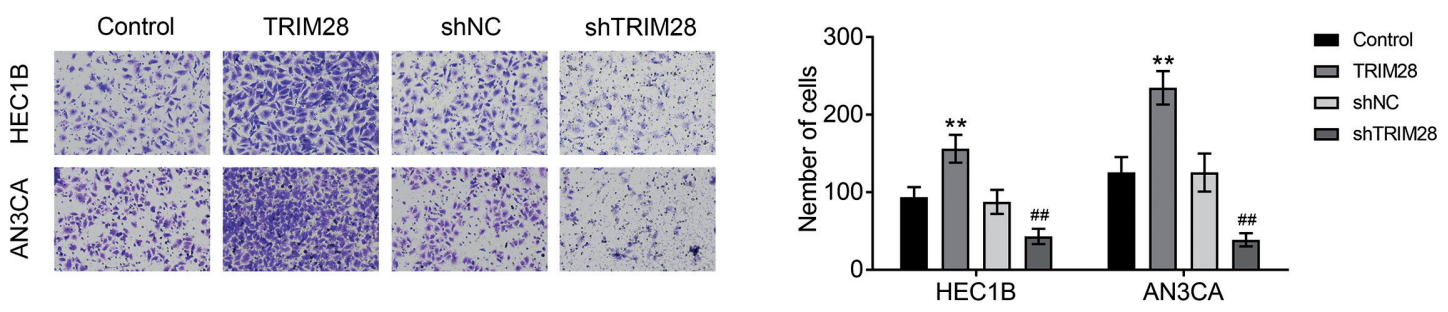

Figure 3. Suppression of TRIM28 inhibited the migration and invasion of EC cells. A. Wound scratch healing assay of HEC1B and AN3CA cell lines after overexpression or knockdown of TRIM28. B. Transwell assay of HEC1B and AN3CA cell lines after overexpression or knockdown of TRIM28. Data were presented as the mean \pm SD with three independent experiments. ${ }^{* *} p<0.01 v s$. Control group; ${ }^{\# \#} p<0.01$ vs. shNC group.

shRNA control (shNC), and two kinds of TRIM28-targeting shRNA (shTRIM28\#1 and shTRIM28\#2), respectively. After transfection, the protein expression level of TRIM28 in HEC1B and AN3CA cell lines were examined. Cells transfected with TRIM28 mimics displayed dramatically higher TRIM28 expression compared to control group. Meanwhile, cells transfected with TRIM28-targeting shRNAs showed strikingly lower TRIM28 expression compared to control group, confirming the successful transfection efficiency (Fig. 2A). TRIM28\#2 was used for TRIM28 knockdown in subsequent experiments due to its better knockdown effect than TRIM28\#1. CCK-8 assay was carried out to detect the effect of TRIM28 on the EC cell proliferation. The results showed that, compared with control group, overexpression of TRIM28 markedly promoted the proliferative ability of EC cell lines, while suppression of TRIM28 expression dramatically inhibited the proliferative ability of EC cell lines (Fig. 2B). Furthermore, clone formation assay was used to evaluate the proliferation of EC cell lines in vitro, and the results revealed that up-regulation of TRIM 28 could increase the number of cell clones and down-regulation of TRIM28 could decrease the number of cell clones (Fig. 2C). These results showed that suppression of TRIM28 could inhibit the proliferation of EC cell.

\section{Suppression of TRIM28 expression inhibited the migration and invasion of EC cells}

Furthermore, to examine the effects of TRIM28 on EC cell migration and invasion, EC cell lines (HEC1B and AN3CA) were transfected with empty vector (Control), TRIM28 mimics (TRIM28), shRNA control (shNC), and TRIM28targeting shRNA, respectively. The wound scratch healing results suggested that the migration of $\mathrm{HEC} 1 \mathrm{~B}$ and AN3CA cells was notably enhanced after TRIM28 was overexpressed, while markedly reduced after transfection of TRIM28targeting shRNA (Fig. 3A). The transwell assays results revealed that the numbers of invaded HEC1B and AN3CA cells were obviously increased after TRIM28 was overexpressed, while dramatically decreased after transfection of TRIM28-targeting shRNA (Fig. 3B). These results suggested that suppression of TRIM28 inhibited the migration and invasion of EC cells. 
TRIM28 was involved in the activation of AKT/mTOR signaling pathway

To estimate whether the mechanism underlying the effects of TRIM28 on promoting the growth of EC cells was mediated by AKT/mTOR pathway, the Western blot assay was applied to detect the expression levels of AKT and mTOR in HEC1B and AN3CA cell lines (Fig. 4). The dramatic upregulation in the phosphorylation levels of AKT and mTOR proteins was found in EC1B and AN3CA cell lines after TRIM28 was overexpressed. However, the markedly downregulation of phosphorylation levels of AKT and mTOR proteins were observed in EC1B and AN3CA cell lines after transfection of TRIM28-targeting shRNA, indicating that TRIM28 could activate AKT and mTOR signaling pathway. These data indicated that TRIM28 was involved in the activation of the $\mathrm{AKT} / \mathrm{mTOR}$ pathway.

\section{Discussion}

Recently, several studies have suggested that TRIM28 has the potential to play an important role in tumor development (Czerwińska et al. 2017). However, TRIM28 is rarely studied in EC, and understanding the molecular regulatory mechanism of TRIM28 in EC may provide the novel therapeutic targets for EC treatment. However, rare studies considered this topic.

TRIM containing proteins have been shown to play vital roles in modulating cell growth and migration processes
(Wei et al. 2018). Many TRIM containing proteins are highly expressed in tissues or organs, contributing to the maintenance of normal tissue functions and homeostasis, and thus affect cell growth and migration (Huang et al. 2017). Moreover, many TRIM containing proteins are implicated in cancer-associated cell growth and migration, and disorders of TRIM containing proteins play a vital role in the occurrence and progression of cancer (Dai et al. 2019). Specifically, studies have also found that TRIM37 promotes the aggressiveness of non-small-cell lung cancer (NSCLC) cells via activating the NF- $\kappa \mathrm{B}$ signaling pathway (Li et al. 2018). Recently, the relationship between TRIM11 and microRNA-5193 in the progression of human prostate cancer was confirmed (Pan et al. 2019). Moreover, TRIM25 was found to promote the invasion and proliferation of colorectal cancer cells via TGF- $\beta$ pathway (Sun et al. 2017). This study disclosed that the mRNA and protein expression level of TRIM28 was higher in EC tissues or cell lines than that in normal tissue or hEEC, respectively. Suppression of TRIM28 inhibited the proliferation, migration, and invasion of EC cells. These results hinted that TRIM28 may act as a promotive factor in the growth and migration of EC cells.

An increasing number of researches confirmed that TRIM containing proteins exert their functions by regulating the expression of target mRNAs (Zhang et al. 2019). A previous study proved that TRIM28 regulates endothelial angiogenic and inflammation activity via retaining protein expression levels of TNFR-1, TNFR-2, and VEGFR2 in endothelial cells
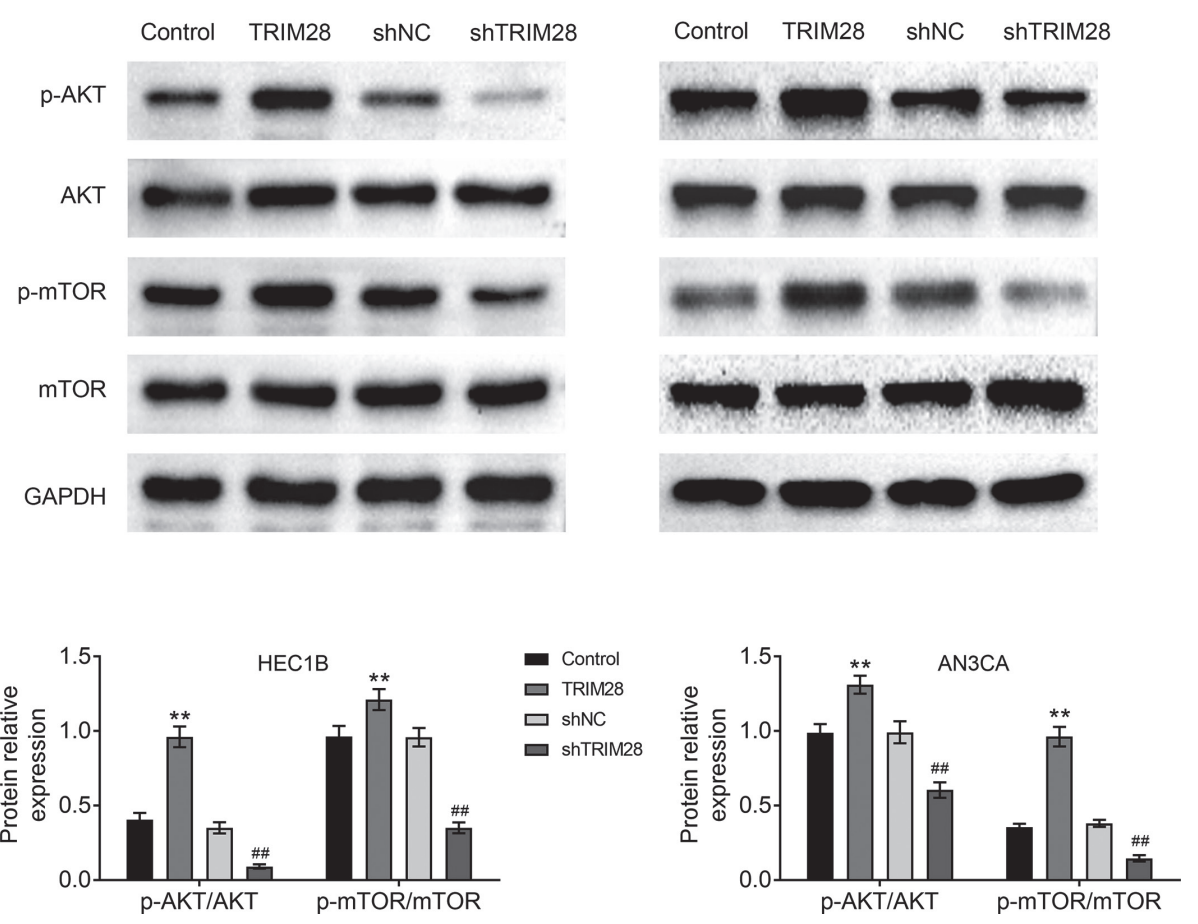

Figure 4. TRIM28 was involved in the activation of AKT/mTOR signaling pathway. The protein levels of AKT, p-AKT, mTOR, and p-mTOR in HEC1B (A) and AN3CA (B) cell lines after overexpression or knockdown of TRIM28, as determined using Western blotting. Relatively quantitative results were determined by ImageJ and shown as histogram. ${ }^{* *} p<0.01$ vs. Control group; ${ }^{\# \#} p<$ 0.01 vs. shNC group. 
(Wang et al. 2017). Moreover, TRIM59 was reported to regulate autophagy via modulating both ubiquitination and transcription of BECN1 protein (Han et al. 2018). In addition, TRIM14 was reported to mediate epithelial-mesenchymal transition via ZEB2 signaling pathway in glioblastoma cells (Feng et al. 2019). Another novel discovery of this study was that AKT and mTOR are the target of TRIM28. AKT belongs to a serine/threonine-specific protein kinase and mTOR belongs to the phosphatidylinositol 3-kinase-related kinase family, which plays a vital role in multiple cellular processes including cell growth, migration, and proliferation. Here, the fact that TRIM28 could target AKT and mTOR was demonstrated by Western blot assays. Overexpression or knockdown of TRIM28 in HEC1B and AN3CA cell lines would promote or inhibit the phosphorylation of AKT and mTOR protein, indicating that TRIM28 promoted the growth and migration of EC cells by regulating the AKT/ mTOR pathway.

In conclusion, in this study, the fact that TRIM28 was overexpressed in EC tissues and cells was discovered. Moreover, suppression of TRIM28 inhibited the proliferation of EC cell. Meanwhile, suppression of TRIM28 inhibited the migration, and invasion of EC cell. Finally, overexpression or knockdown of TRIM28 in HEC1B and AN3CA cell lines would promote or inhibit the phosphorylation of AKT and mTOR protein. All these results figured out the role of TRIM28/AKT/mTOR signaling pathway in promoting the growth and migration of EC cells, which could conceivably pave the path for advanced therapeutic targets in EC.

Conflict of interest. The authors state that there are no conflicts of interest to disclose.

Ethics approval. Ethical approval was obtained from the Ethics Committee of the First Affiliated Hospital of Gannan Medical College.

Statement of informed consent. Written informed consent was obtained from a legally authorized representative(s) for anonymized patient information to be published in this article.

Authors' contributions. YCh and HCh designed the study, supervised the data collection, HL analyzed the data, interpreted the data, YCh, HCh and HL prepared the manuscript for publication and reviewed the draft of the manuscript. All authors have read and approved the manuscript.

\section{References}

Buhtoiarova TN, Brenner CA, Singh M (2016): Endometrial carcinoma: role of current and emerging biomarkers in resolving persistent clinical dilemmas. Am. J. Clin. Pathol. 145, 8-21 https://doi.org/10.1093/ajcp/aqv014
Czerwińska P, Mazurek S, Wiznerowicz M (2017): The complexity of TRIM28 contribution to cancer. J. Biomed. Sci. 24, 63 https://doi.org/10.1186/s12929-017-0374-4

Dai X, Geng F, Li M, Liu M (2019): Tripartite motif-containing 11 regulates the proliferation and apoptosis of breast cancer cells. Oncol. Rep. 41, 2567-2574 https://doi.org/10.3892/or.2019.7015

Deng B, Zhang S, Zhang Y, Miao Y, Meng X, Guo K (2017): Knockdown of Tripartite Motif Containing 28 suppresses the migration, invasion and epithelial-mesenchymal transition in ovarian carcinoma cells through down-regulation of Wnt/ $\beta$ catenin signaling pathway. Neoplasma 64, 893-900 https://doi.org/10.4149/neo_2017_611

Feng S, Cai X, Li Y, Jian X, Zhang L, Li B (2019): Tripartite motifcontaining 14 (TRIM14) promotes epithelial-mesenchymal transition via ZEB2 in glioblastoma cells. J. Exp. Clin. Cancer Res. 38, 57 https://doi.org/10.1186/s13046-019-1070-X

Han T, Guo M, Gan M, Yu B, Tian X, Wang JB (2018): TRIM59 regulates autophagy through modulating both the transcription and the ubiquitination of BECN1. Autophagy 14, 2035-2048 https://doi.org/10.1080/15548627.2018.1491493

Huang XQ, Zhang XF, Xia JH, Chao J, Pan QZ, Zhao JJ, Zhou ZQ, Chen CL, Tang Y, Weng DS, et al. (2017): Tripartite motifcontaining 3 (TRIM3) inhibits tumor growth and metastasis of liver cancer. Chin. J. Cancer 36, 77 https://doi.org/10.1186/s40880-017-0240-5

Li F, Wang Z, Lu G (2018): TRIM28 promotes cervical cancer growth through the mTOR signaling pathway. Oncol. Rep. 39, $1860-1866$ https://doi.org/10.3892/or.2018.6235

Li Y, Deng L, Zhao X, Li B, Ren D, Yu L, Pan H, Gong Q, Song L, Zhou X, Dai T (2018): Tripartite motif-containing 37 (TRIM37) promotes the aggressiveness of non-small-cell lung cancer cells by activating the NF- $\kappa B$ pathway. J. Pathol. 246, 366-378 https://doi.org/10.1002/path.5144

Liu B, Li X, Liu F, Li F, Wei S, Liu J, Lv Y (2019): Expression and significance of TRIM 28 in squamous carcinoma of esophagus. Pathol. Oncol. Res. 25, 1645-1652 https://doi.org/10.1007/s12253-018-0558-6

Miller KD, Nogueira L, Mariotto AB, Rowland JH, Yabroff KR, Alfano CM, Jemal A, Kramer JL, Siegel RL (2019): Cancer treatment and survivorship statistics, 2019. CA Cancer J. Clin. 69, 363-385 https://doi.org/10.3322/caac.21565

Pan Y, Zhang R, Chen H, Chen W, Wu K, Lv J (2019): Expression of tripartite motif-containing proteactiin 11 (TRIM11) is associated with the progression of human prostate cancer and is downregulated by microRNA-5193. Med. Sci. Monit. 25, 98-106 https://doi.org/10.12659/MSM.911818

Samat AHA, Embong H, Harunarashid H, Maskon O (2020): Predicting ventricular arrhythmias and in-hospital mortality in acute coronary syndrome patients presenting to the emergency department. Signa Vitae 16, 55-64 https://doi.org/10.22514/sv.2020.16.0008

Su C, Li H, Gao W (2018): TRIM28 is overexpressed in glioma and associated with tumor progression. Onco. Targets Ther. 11, 6447-6458 https://doi.org/10.2147/OTT.S168630 
Sun N, Xue Y, Dai T, Li X, Zheng N (2017): Tripartite motif containing 25 promotes proliferation and invasion of colorectal cancer cells through TGF- $\beta$ signaling. Biosci. Rep. 37, BSR20170805 https://doi.org/10.1042/BSR20170805

van Gent M, Sparrer KMJ, Gack MU (2018): TRIM proteins and their roles in antiviral host defenses. Annu. Rev. Virol. 5, 385-405 https://doi.org/10.1146/annurev-virology-092917-043323

Wang Y, Li J, Huang Y, Dai X, Liu Y, Liu Z, Wang Y, Wang N, Zhang $P$ (2017): Tripartite motif-containing 28 bridges endothelial inflammation and angiogenic activity by retaining expression of TNFR-1 and -2 and VEGFR2 in endothelial cells. FASEB J. 31, 2026-2036 https://doi.org/10.1096/fi.201600988RR

Wei C, Cheng J, Zhou B, Zhu L, Khan MA, He T, Zhou S, He J, Lu X, Chen H, et al. (2016): Tripartite motif containing 28 (TRIM28) promotes breast cancer metastasis by stabilizing TWIST1 protein. Sci. Rep. 6, 29822 https://doi.org/10.1038/srep29822

Wei Y, Chen S, Wang M, Cheng A (2018): Tripartite motif-containing proteins precisely and positively affect host antiviral immune response. Scand. J. Immunol. 87, e12669 https://doi.org/10.1111/sji.12669
World Medical Association Declaration of Helsinki (2013): Ethical principles for medical research involving human subjects. JAMA 310, 2191-2194 https://doi.org/10.1001/jama.2013.281053

Yin J, Kim TH, Park N, Shin D, Choi HI, Cho S, Park JB, Kim JH (2016): TRIM71 suppresses tumorigenesis via modulation of Lin28B-let-7-HMGA2 signaling. Oncotarget 7, 79854-79868 https://doi.org/10.18632/oncotarget.13036

Yokoe T, Toiyama Y, Okugawa Y, Tanaka K, Ohi M, Inoue Y, Mohri Y, Miki C, Kusunoki M (2010): KAP1 is associated with peritoneal carcinomatosis in gastric cancer. Ann. Surg. Oncol. 17, 821-828 https://doi.org/10.1245/s10434-009-0795-8

Zhang P, Zhang H, Wang Y, Zhang P, Qi Y (2019): Tripartite motifcontaining protein 59 (TRIM59) promotes epithelial ovarian cancer progression via the focal adhesion kinase (FAK)/AKT/ Matrix Metalloproteinase (MMP) pathway. Med. Sci. Monit. 25, 3366-3373 https://doi.org/10.12659/MSM.916299

Received: December 3, 2020

Final version accepted: March 22, 2021 